\title{
The Upgraded Calibration System for the Scintillator-PMT Tile Hadronic Calorimeter of the ATLAS experiment at CERN/LHC
}

\author{
Dhiman Chakraborty ${ }^{1}$ \\ Physics Department, Northern Illinois University, DeKalb, IL 60115, USA
}

\begin{abstract}
The TileCal section of the hadronic calorimeter of the ATLAS detector at the Large Hadron Collider (LHC), CERN, is a sampling calorimeter employing steel plates as absorber and plastic scintillator tiles read out by photomultiplier tubes (PMTs) as the active medium. We present an overview of the calibration system that enables the TileCal to meet its design goals of sub-1\% level accuracy as well as excellent short- and long-term stability through constant monitoring.
\end{abstract}

\section{The ATLAS TileCal}

Run 2 of the LHC has been delivering proton-proton, proton-ion, and ionion collisions at the highest-ever energies since 2015. ATLAS, one of four detectors to study the outcome of such collisions, has been collecting data smoothly 5 through its many subsystems working in tandem. The TileCal is the central section of the hadronic calorimeter of the ATLAS experiment [1]. It provides important information for reconstruction of hadrons, jets, hadronic decays of tau leptons and missing transverse energy in collision events. The light produced by the passage of charged particles through the active medium is collected and transmitted by wavelength shifting (WLS) fibres to PMTs located on the outside of the calorimeter. The active volume is segmented longitudinally and transversally into about 5000 cells, each read out by two PMTs. Figure 1 shows

\footnotetext{
${ }^{1}$ Corresponding author, on behalf of the ATLAS Tile Calorimeter System.
} 
a drawing of one of 64 modules in each of four sections of the ATLAS TileCal. Hydraulic tubes carry movable capsules containing a radioactive source for scintillator calibration.

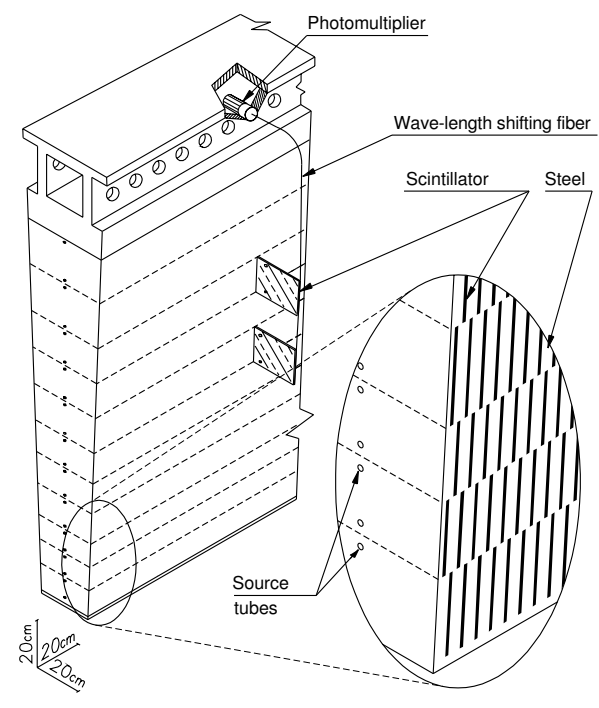

Figure 1: An ATLAS TileCal module.

\section{Calibration of the TileCal}

An elaborate multi-component system is used to calibrate and monitor the stability and performance of each part of the TileCal readout chain 2. This system, which comprises ${ }^{137} \mathrm{Cs}$ radioactive sources, laser and charge injection elements, allows us to monitor, and ensure uniformity of, the cell response at each stage of the signal processing - from scintillation to digitization. A schematic representation of this calibration chain is shown in Figure 2 .

The energy $E$, in units of $\mathrm{MeV}$, deposited in a scintillator cell is estimated using the equation

$$
E[\mathrm{MeV}]=A[\mathrm{ADC}] \times C_{\mathrm{CIS}}\left[\frac{\mathrm{pC}}{\mathrm{ADC}}\right] \times C_{\mathrm{TB}}\left[\frac{\mathrm{MeV}}{\mathrm{pC}}\right] \times C_{\mathrm{Laser}} \times C_{\mathrm{Cs}},
$$

${ }_{25}$ where $A$ is the $\mathrm{ADC}$ count read out, $C_{\mathrm{CIS}}$ is the $\mathrm{ADC}$ count per $\mathrm{pC}$ of charge collected by the PMT (CIS stands for "Charge Injection System"), $C_{\mathrm{TB}}$ is the 


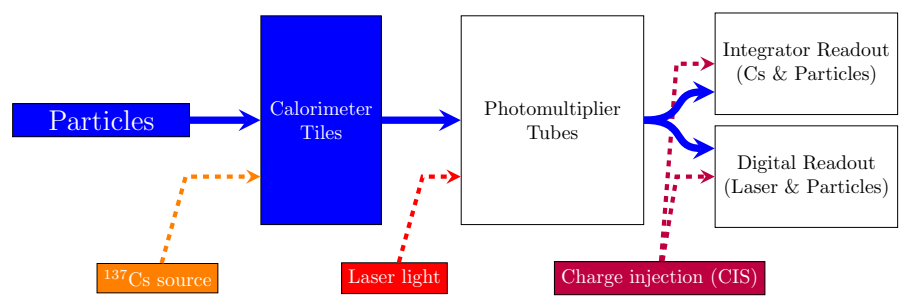

Figure 2: The calibration chain for ATLAS TileCal.

pC-to-MeV ratio measured using test beams, $C_{\text {Laser }}$ is the calibration constant for the PMT response to light generated in the scintillator tile, and $C_{\mathrm{Cs}}$ for the cell's response to the radiation from the ${ }^{137} \mathrm{Cs}$ radioactive source.

\section{3. The Calibration System}

Laser, Charge Injection, and Pedestal calibration runs allow monitoring of timing, stability, and noise, while Cs runs help calibrate the entire optical path from scintillator tiles to WLS fibres down to integrated pulses from the PMTs. Approximately $11 \%$ of the 192 TileCal modules were calibrated using test beams and the EM scale of $1.05 \mathrm{pC} / \mathrm{GeV}$ was transferred to the full detector using the calibration system. Independent assessment of calibration runs from a data quality perspective serves as a cross check for any problems that may arise during physics runs.

Cs: $\mathrm{A}{ }^{137} \mathrm{Cs} \gamma$ source $\left(10 \mathrm{mCi}, t_{\frac{1}{2}}=30 \mathrm{yr}\right)$ provides, with a precision better 40 than $0.3 \%$, the $\mathrm{pC} \rightarrow \mathrm{MeV}$ calibration for PMT gains, which are adjusted accordingly by tuning the high voltage (HV) to ensure uniformity of cell response. Normally, a full scan, which takes 8 hours, is performed once a month.

Laser: A laser system delivers $532 \mathrm{~nm}$ (green) light through 400 clear fibres to all PMTs. The optics box and control electronics were upgraded for Run

45 2. The system provides corrections for timing jumps and PMT gain drifts between Cs scans. These are further validated against gains seen in the Minimum Bias Integrator system, which measures PMT currents integrated over $\sim 10 \mathrm{~ms}$. 
Laser calibration, taken twice a week, helps isolate problems with HV stability and charge injection in bad channels. The precision of the gain variation measurement is better than $0.5 \%$.

Charge Injection: Known amounts of electric charge are injected directly into the integrator and digital readout in pulses of varying shape (amplitude) to calibrate ADC response. The injection timing with respect to the ADC clock can be controlled, which allows modeling of physics pulses from the PMTs. The calibration only takes a few minutes, and is performed twice a week. Typically, the constants, whose systematic uncertainty is $\sim 0.7 \%$, need to be updated no more than once a month. The average stability over all of 2016 was $0.04 \%$ (rms).

\section{Performance of the Calibration System}

Based on Run 1 experience (2010-2013), the TileCal calibration systems were improved for Run 2. The current performance meets the design goals of sub-1\% level accuracy and excellent stability [2, 3].

\section{Acknowledgement}

The author would like to thank the U.S. National Science Foundation for supporting his research activities.

\section{References}

[1] ATLAS TileCal: Technical Design Report, ATLAS, CERN, Geneva, 1996. URL https : //cds . cern. ch/record/331062

[2] O. Solovyanov, Calibration and Performance of the ATLAS Tile Calorimeter, Tech. Rep. ATL-TILECAL-PROC-2017-010, CERN, Geneva (2017). URL https://cds . cern . ch/record/2271565

[3] ATLAS Experiment - Public Results - Approved Tile Calorimeter Plots. 【 URL https://twiki.cern.ch/twiki/bin/view/AtlasPublic/ ApprovedPlotsTile 